\title{
Simple Method of Economical Analysis of Diagnosis Procedure (Used in Screening of Thyroid Gland Diseases in Pregnant Women)
}

\section{Z. Telička', J. Jiskra', D. Springer², Z. Límanová1}

'Third Department of Medicine, General University Hospital and First Faculty of Medicine, Charles University in Prague, Czech Republic ${ }^{2}$ Department of Clinical Biochemistry and Laboratory Medicine, General Teaching Hospital, First Faculty of Medicine Charles University Supervisor: Prof. MUDr. Štěpán Svačina, DrSc., MBA

\section{Summary}

Thyroid gland diseases are frequent in pregnant women. In the Czech Republic, there is not a screening for this disease. Our objective was to evaluate the initial cost of the study of screening of thyroid gland disease in pregnant women.

Methods: We used simple statistical methods for comparing the cost of the study (examination of TSH and TPOAb) with the costs and efficiency when TSHonly or TPOAb-only screening would be performed.

Screening of TSH only is 2.7 times costeffective than screening of TPOAb. Screening of TSH only may lead to miss 77 patients with TPOAb positive results and screening of TPOAb only may lead to miss 77 patients with TSH positive results.

Discussion: To achieve better sensitivity, it is necessary to perform screening of TSH and TPOAb together.

Keywords: thyroid gland disease, thyreopathy, pregnancy, cost-effectivenes, screening, iq, qaly

\section{Introduction}

Thyroid gland diseases are frequent in pregnant women [1]. The untreated dysfunction of the thyroid gland during the pregnancy may have a negative impact both to a mother and mostly to a child. It can cause low concentrations of thyroid gland hormones which are necessary for the proper development of the child brain, especially during the beginning of pregnancy [2]. Screening tests for thyroid disease consist of measurements of thyroid peroxidase antibodies (TPOAb), thyroid stimulating hormone (TSH) levels and levels of thyroid hormones (T3 and T4) in the blood. Anti-TPO antibodies indicate an autoimmune reaction in which the body sends immune cells to attack the thyroid gland. In foreign studies, about $11 \%$ of them have proven the disease after a blood examination [1]. Observational studies have concluded that the treatment of maternal hypothyroidism with the thyroid hormone reduces the incidence of gestational hypertension in the mother [3] and improves IQ in the child [4], [5]. Positive TSH results (and negative TPOAb) may lead to improper development of child's brain during the pregnancy and positive TPOAb results (and negative TSH) may lead to the post partum thyroid disease. High ratio of incidence makes clinicians to introduce a systematic screening for this disease. Currently, there is an ongoing discussion whether to establish the screening targeted to all pregnant women or to women with a risk factor and what parameters to examine. All these factors are influenced by the economical aspect from two sides:

1)Is the cost for screening adequate to a number of positive matched examinations?

2)What benefits does it bring to a mother and a child the successfully treated thyroid gland disease after the positively matched examination?

\subsection{Already performed foreign study}

The cost effectiveness analysis was already performed on screening of pregnant women in USA. Three strategies were used on the tested group: Women with no screening; one-time screening of TSH and one-time screening of TPOAb. Abnormal screening tests were followed by further testing and hypothyroid women were treated by thyroxine until the test results came to the normal level. Than the cost of the effectiveness analysis was performed, it included the cost of:

- visits at clinic and endocrinologist,

- laboratory screening tests for TSH or TPOAb,

- levothyroxine treatment.
In the study the cost of a low IQ was estimated. Authors of the project worked with consideration, that there is a significant fall of child's $I Q$ if a mother is not treated during the early state of pregnancy. A one-point difference in $I Q$ is associated with about a $2 \%$ difference in earnings [7]. With this assumption they found a loss of USD 15513 in future earnings per IQ point. Based on the follow-up to the Haddow study [8] they assumed that children of untreated overtly and subclinically hypothyroid mothers have on average a 9.2- and 4.2-point decrement in IQ (after adjusting by 0.8 point for differences in socioeconomic status compared with controls). By developing the Markov Model [9] it was possible to find out the Incremental cost-effectiveness ratio for the child per quality adjusted life year (QALY) if the woman is treated for the thyroid gland disease during the pregnancy compared to [6]. Screening pregnant women in the first trimester with TSH was cost-saving compared with no screening, while screening with TPOAb was cost-effective compared with TSH screening at USD 15 182/QALY.

\subsection{Initial study performed in the Czech Republic}

A multicentre pilot study has started in 13 regions of the Czech Republic supported by the General Health Insurance Company focused on examinations of thyroid gland diseases in pregnant women. The aim of this study was to find out initial data which could help the clinicians and the insurance company with the decision, whether the screening of women could be beneficial to women and cost-effective for the insurance system. 
In our study we tried to find out the costs of this initial study compared to positivelyscreened tests and simulate the costs in the current situation when the screening is not paid by the insurances companies. We also simulated the costs in the situation when the screening could contain only the TSH or only the TPOAb serum tests.

\section{Methods}

During the 11-13 week of pregnancy, pregnant women were tested for TSH and TPOAb. If the laboratory reported positive results, women were recommended to visit the endocrinologist who eventually started the treatment. Current costs in the insurance system for testing of these three results in the standard test cost $518 \mathrm{CZK}$ (TSH 140 CZK; TPOAb 378 CZK). Since there it is not a standardized method for TSH and TPOAb, each laboratory has developed its own test methods and ranges. In our study we used normal levels used in each laboratory. Orientation normal levels for TSH are 0.1-3.7 mU/l and for TPOAb $\leq 34 \mathrm{IU} / \mathrm{ml}$. From the study all the patients positively screened due to the suppressed TSH were removed to exclude healthy women with physiologic THS suppression in the first trimester of pregnancy. Therefore we analyzed only the patients with elevated TSH $(>3.7$ $\mathrm{mIU} / \mathrm{II}$. We calculated the summary cost for the initial screening and according to the number of positive results of tested parameters we specified the cost of one positive diagnosis.

\section{Results}

The costs for TSH and TPOAb screening was 1373218 CZK. 612 (23\%) patients were tested positive in one of these two parameters. It means the cost for one positive result of $2243 \mathrm{CZK}$.

We tested if it would be sufficient to test women for TSH or TPOAb only. Positively tested (up of reference range) for TSH were found 269 women $(10.14 \%$ ) and from this $77(28.63 \%)$ where TPOAb positive and $192(71.37 \%)$ were TPOAb negative. The cost for this screening would be 371140 CZK; 1380 CZK for one TSHpositive screening. But 2382 (89.85\%) TSH-screening negative women have 143 (6\%) TPOAb positive results. It means that TSH-only screening may lead to miss 77 patients with TPOAb positive results.
Positively tested for TPOAb-screening only were $220(8.3 \%)$ women and from this 77 (35\%) have TSH positive results. The cost would be 1002078 CZK; 4555 CZK for one TPOAb-positive screening. But $2431(91.70 \%)$ TPOAb negative women have from this $192(7.9 \%)$ TSH positive screening results. It means that TPOAbonly screening may lead to miss 77 patients with TSH positive results.

\section{Discussion}

From the results we have found out that both TSH and TPOAb screening tests are not substitutable and they have to be screened together although the costs for the TSH screening is 2.7 times most costeffective than TPOAb screening. Screening of one of the above parameters could mean lost of 77 positive women.

Additionally, screening of these two parameters is important due to fact that positive results of each parameter mean for the mother and child a different risk: Positive TSH can cause improper development of child's brain during the pregnancy and positive TPOAb results can cause the post partum thyroid disease.

\section{Acknowledgements}

This work was supported by a grant from the Czech Health Ministry IGA NS10595-3 and by the project SVV-2010-265513.

\section{References}

[1] Hollowell J.G., Staehling N.W., Flanders W.D., Hannon W.H., Gunter E.W., Spencer C.A., Braverman L.E.: Serum TSH, T(4), and thyroid antibodies in the United States population (1988 to 1994): National Health and Nutrition Examination Survey (NHANES III). J Clin Endocrinol Metab. 2002 Feb;87(2):489-99.

[2] Springer D., Zima T., Limanova Z.: Reference intervals in evaluation of maternal thyroid function during the first trimester of pregnancy. Eur J Endocrinol. 2009 May;160(5):791-7. Epub 2009 Feb 19.

[3] Leung A.S., Millar L.K., Koonings P.P., Montoro M., Mestman J.H.: Perinatal outcome in hypothyroid pregnancies. Obstet Gynecol. 1993 Mar;81(3):349-53.

[4] Haddow J.E., Palomaki G.E., Allan W.C., Williams J.R., Knight G.J., Gagnon J., O'Heir C.E., Mitchell M.L., Hermos R.J., Waisbren S.E., Faix J.D., Klein R.Z:. Maternal thyroid deficiency during pregnancy and subsequent neuropsychological development of the child. N Engl J Med. 1999 Aug 19;341(8):549-55.
[5] Man E.B., Brown J.F., Serunian S.A:. Maternal hypothyroxinemia: psychoneurological deficits of progeny. Ann Clin Lab Sci. 1991 Jul-Aug;21(4):227-39.

[6] Dosiou C., Sanders G.D., Araki S.S., Crapo L.M.: Screening pregnant women for autoimmune thyroid disease: a costeffectiveness analysis. Eur J Endocrinol. 2008 Jun; 158(6):841-51.

[7] Grosse S.D., Matte T.D., Schwartz J., Jackson R.J.: Economic gains resulting from the reduction in children's exposure to lead in the United States. Environ Health Perspect. 2002 Jun;110(6):563-9.

[8] Klein R.Z., Sargent J.D., Larsen P.R., Waisbren S.E., Haddow J.E., Mitchell M.L. Relation of severity of maternal hypothyroidism to cognitive development of offspring. J Med Screen. 2001;8(1):18-20.

[9] Sonnenberg F.A., Beck J.R.: Markov models in medical decision making: a practical guide. Med Decis Making. 1993 OctDec;13(4):322-38.

\section{Contact}

Mgr. Zdeněk Telička

Third Department of Medicine,

General University Hospital and First

Faculty of Medicine, Charles University

Prague,

U Nemocnice 1

12808 Prague 2

Czech Republic

e-mail: zdenek@telicka.cz 